\title{
PREVISIÓN DE NECESIDADES FORMATIVAS PARA UN ENVEJECIMIENTO ACTIVO
}

\section{ACTIVE AGEING TRAINING" NEEDS FOR SPANISH POPULATION 45 TO 54 YEARS OLD}

\author{
Dr. Antonio Matas-Terrón ${ }^{1}$ \\ amatas@uma.es \\ Dr. Juan José Leiva Olivencia ${ }^{2}$ \\ jjleiva@uma.es \\ Pablo Daniel Franco Caballero ${ }^{1}$ \\ pablo.franco.caballero@gmail.com
}

\begin{abstract}
${ }^{1}$ Universidad de Málaga. Facultad de Ciencias de la Educación. Departamento de Métodos de Investigación e Innovación Educativa. Bulevar Louis Pasteur s/n 29010 Málaga (España) ${ }^{2}$ Universidad de Málaga. Facultad de Ciencias de la Educación. Departamento de Didáctica y Organización Escolar. Bulevar Louis Pasteur s/n 29010 Málaga (España)
\end{abstract}

Las nuevas tecnologías (NT) cada vez se usan más para tener una vida saludable. El objetivo del estudio es analizar el grado de penetración de las NT en la población española de 45 a 54 años para estimar su utilización en un envejecimiento saludable. Se han utilizado los datos de la encuesta sobre equipamientos en los hogares españoles del 2006 al 2013 (Instituto Nacional de Estadística). La previsión se ha llevado a cabo mediante una metodología Delphi. Los datos se han analizado descriptiva y cualitativamente. Los resultados sugieren que la población debe implicarse en su propio plan de formación.

Palabras clave: Nuevas tecnologías, envejecimiento, alfabetización, educación permanente.

Ever more, new technologies (NT) are used to have a healthy ageing. The aim was to analyze presence of NT in Spanish population from 45 to 54 years old. The need to develop specific training programs in NT was analyzed with a Delphi methodology. We used National Statistics Institute data, in 2006 to 2013 period, about Equipment in Spanish Homes. Results indicate that population need to involve themselves to get a healthy ageing.

Keywords: New technologies (NT), ageing, literacy, lifelong learning. 


\section{Introducción.}

Las personas mayores son un colectivo de especial relevancia como destinatarias de políticas concretas de bienestar social donde la educación, los servicios sociales, la dependencia y la salud son ejes básicos de una política inclusiva y global (González, Gómez \& Mata, 2012). Este colectivo exige iniciativas formativas y sociales que ayuden a generar un envejecimiento activo que esté construido sobre las sólidas bases de iniciativas y actividades donde el conocimiento científico y tecnológico suscita sinergias francamente positivas y enriquecedoras (Punie \& Ala-Mutka, 2007). En los últimos años, algunos países están interesados en incrementar las competencias digitales de los adultos por el carácter preventivo e integral que tiene su uso y disfrute de cara a un envejecimiento activo (Castaño, Redecker, Vuorikari \& Punie, 2013).

Un claro ejemplo de ello se puede observar en Suecia y también en los países bálticos donde se vienen implementando medidas e iniciativas de educación digital para personas mayores, vinculándolas al ámbito asociativo, educativo y cultural (Berner, Rennemark, Jogréus \& Berglund, 2012; Grzesiak \& Richert-Ka ̈̈mierska, 2013; Berner, Rennemark, Jogréus \& Berglund, 2013).

Los estudios sobre TIC y personas mayores han llegado a una serie de conclusiones, de la cuales se quiere destacar las siguientes:

La penetración de las TIC es cada vez mayor más en los hogares españoles, tal como queda patente en el Informe Recopilatorio 2004-2012, «las TIC en los Hogares Españoles» publicado por el Observatorio Nacional de las Telecomunicaciones y la Sociedad de la Información (Ontsi, 2013).
En los estudios revisados queda patente una desigualdad de género en relación al uso y utilidad que le dan hombres y mujeres mayores a las TIC. Así, el último informe del Observatorio para las Personas Mayores del Ministerio de Sanidad, Servicios Sociales e Igualdad del Gobierno de España (IMSERSO, 2012) indicaba que las mujeres tienen un menor nivel de formación que los hombres, y que esta situación se relaciona con el empleo de las TIC en su vida cotidiana. Este informe incide en una mayor necesidad de alfabetización mediática, pero considera un hándicap difícil de superar para el uso del ordenador e internet la falta de formación suficiente para enfrentarse a las nuevas tecnologías. Según este informe existe un $68.2 \%$ de personas mayores (65 a 74 años) que declara utilizar el teléfono móvil, un $22.4 \%$ que ha utilizado alguna vez el ordenador, un $16.4 \%$ que ha utilizado internet y un $4.5 \%$ que alguna vez ha comprado a través de esta vía.

Los mayores no quieren quedarse al margen de los avances. Manifiestan interés por la tecnología y el aprendizaje en general, y especialmente en las TIC (Sebastián \& Martínez, 2013).

Aunque pueden presentar signos de ansiedad en los primeros momentos del uso de las TIC, son perseverantes hasta conseguir comprender su funcionamiento (Aldana, García \& Jacobo, 2012; Tam, 2014b).

Las habilidades que practican con las TIC promueven la independencia y la calidad de vida (Luque, 2007; Tam, 2013, 2014a).

En un futuro inmediato, y teniendo en cuenta la presencia creciente de las TIC en los hogares, así como su rápido desarrollo, es previsible que las personas mayores utilicen la tecnología para mantener una vida activa y productiva en todos los sentidos, tal como ya se apunta en algunos estudios y 
ensayos (Rosenberg et al., 2010; Burmeister, 2010; Shubert, 2010).

Para la utilización de esta tecnología futura es necesario que las personas mantengan una actitud positiva hacia el aprendizaje a lo largo de la vida (ALV). En este sentido, la tecnología no será solamente un medio para el ALV tal como se ha puesto de relieve en distintos estudios (Regan, 1998; Salanova \& Peiró, 2001; García Aretio, 2007; Ortoll, 2007), sino que es necesario desarrollar el ALV para usar dicha tecnología. Esta idea ha sido tratada en la última década bajo el concepto de alfabetización digital:

Alfabetización digital es una propuesta indispensable de formación para el ser humano, y permite (más allá de la pura obtención de conocimientos, competencia, capacidades, destrezas o habilidades para el manejo operativo de mecanismos, instrumentos o lenguajes tecnológicos de comunicación digital) la posibilidad de entender y generar mensajes comunicativos, reconociendo el contexto social donde se ubica y desde una creativa posición ciudadana reflexiva y crítica (Ojeda, 2008, p. 85).

\section{Método.}

\subsection{Procedimiento.}

Esta investigación se ha diseñado combinando la metodología Delphi con los resultados de la encuesta sobre uso de las TIC en los hogares procedentes del Instituto Nacional de Estadística (INE). Por tanto, se trata de un diseño cualitativo apoyado sobre una investigación externa basada en encuestas y cuya finalidad es predictiva.

En una primera fase se recogió la información que el INE ofrece sobre sus
«Encuestas sobre equipamiento y uso de Tecnologías de la Información y la Comunicación en los hogares» (ETIC) desde el año 2006 al 2013.

Posteriormente se aplicó la metodología Delphi. En esta fase se enviaron los resultados de dos ítems (ver más abajo) de las ETIC a un panel de expertos para que valorasen dichos datos. Este procedimiento se desarrolló entre el diciembre de 2013 y mayo de 2014 utilizando los datos publicados por el INE en diciembre de 2013.

\subsection{Datos del Instituto Nacional de Estadística.}

La fuente de datos fueron las ETIC realizadas desde el año 2006 hasta el 2013 por el Instituto Nacional de Estadística . Estos datos son públicos a través de la página web del INE y se autoriza su utilización para estudios como el que aquí se presentan. Se eligió este periodo de años porque durante el mismo los cuestionarios de las ETIC han experimentado variaciones menores en su redacción, facilitando la comparación de resultados entre años para cada ítem.

Estas encuestas se realizan a través de un procedimiento probabilístico que garantiza la representatividad de la muestra con relación al ámbito nacional. La base de datos contiene información que van desde las 23371 viviendas encuestadas en 2006 a las 15820 de 2013. En la Tabla 1 se exponen las secciones censales usadas para las encuestas en cada año, así como el número total de viviendas encuestadas.

Las encuestas realizadas registraron datos de los residentes en las viviendas, de forma que el número total de personas sobre las que se tienen datos oscila entre los 5813460 ciudadanos de 2006 y los 6937884 de 2013. 
Total viviendas encuestadas

Año Secciones censales

\begin{tabular}{lll}
\hline 2013 & 2500 & 15820 \\
2012 & 2530 & 15965 \\
2011 & $2919 *$ & 18834 \\
2010 & $3005^{*}$ & 19384 \\
2009 & $3045^{*}$ & 19644 \\
2008 & 3140 & 20502 \\
2007 & 3140 & 25145 \\
2006 & 3060 & 23371 \\
\hline
\end{tabular}

Tabla 1. Secciones censales y encuestas realizadas desde 2006 a 2013 (muestra). Leyenda: * Estimadas a partir del total de viviendas encuestadas.

En esta investigación solamente se tuvieron en cuenta los datos correspondientes al segmento de población situados entre los 45 a 54 años, que corresponde con el grupo de ciudadanos que en los próximos 20 años se considerarán dentro del grupo de personas mayores. En la Tabla 2 se indica el número total de la población de este segmento de edad sobre las que se ha recogido los datos.

\subsection{Indicadores utilizados en la encuesta del INE.}

El conjunto de ítems de la encuesta de INE fue sometido a un proceso de análisis por parte de un grupo de 4 docentes e investigadores en el área de Métodos de Investigación. El objetivo de este análisis era concretar qué ítems podrían servir mejor como indicadores útiles para la investigación. El análisis se llevó a cabo a través de un grupo de discusión, con una duración de tres horas.

Del conjunto de ítems que han conformado los distintos cuestionarios del INE, se han elegido dos ítems específicos que se han ido repitiendo en toda la serie de años (2006 a 2013):

- Ítem: «Personas que han utilizado Internet al menos una vez por semana en los últimos 3 meses». Este ítem ha sido elegido porque el acceso a internet supone que la persona es competente en el uso de los equipos informáticos y porque el uso de «al 


\begin{tabular}{llll}
\hline Año & Total & Hombres & Mujeres \\
\hline 2006 & 5813160 & 2890748 & 2922412 \\
2007 & 5918212 & 2944403 & 2973809 \\
2008 & 6164279 & 3068096 & 3096183 \\
2009 & 6297064 & 3135472 & 3161592 \\
2010 & 6439617 & 3205099 & 3234518 \\
2011 & 6595315 & 3282944 & 3312371 \\
2012 & 6715601 & 3342186 & 3373415 \\
2013 & 6937884 & 3471940 & 3465944 \\
\hline
\end{tabular}

Tabla 2. Estimación número de personas de 45 a 54 años de edad de la población origen.

menos una vez por semana» es un indicador de frecuencia.

Ítem: «Personas que han comprado a través de internet en los últimos 3 meses». Este ítem es de interés porque indica un manejo avanzado de las herramientas que proporciona Internet.

\subsection{Metodología Delphi.}

Esta metodología es una variante de los grupos nominales donde un grupo de expertos (panel) realizan una valoración sobre el futuro desarrollo de un fenómeno (Tójar, 2006). Se trata de un procedimiento subjetivo basado en la opinión de dichos expertos, que se utiliza para la prospección de acontecimientos cuando no es viable aplicar otros métodos más objetivos. Su objetivo es la consecución de un consenso basado en la discusión entre expertos. Es un proceso repetitivo que se inicia con la elaboración de un cuestionario que ha de ser contestado por los expertos. Una vez recibidas las contestaciones al primer cuestionario, se vuelve a realizar y enviar otro cuestionario basado en el anterior donde se pregunta sobre las primeras contestaciones de los mismos expertos. Finalmente los responsables del estudio extraen las conclusiones a partir de la explotación estadística de los datos obtenidos, y del análisis de contenido de las contestaciones. El proceso se enriquece con una triangulación entre juecesinvestigadores para garantizar la calidad de los resultados.

La metodología Delphi se organiza de tal forma que las opiniones de los expertos son

Píxel-Bit. Revista de Medios y Educación. No 48 Enero 2016. ISSN: 1133-8482. e-ISSN: 2171-7966. doi: http://dx.doi.org/10.12795/pixelbit.2016.i48.15 


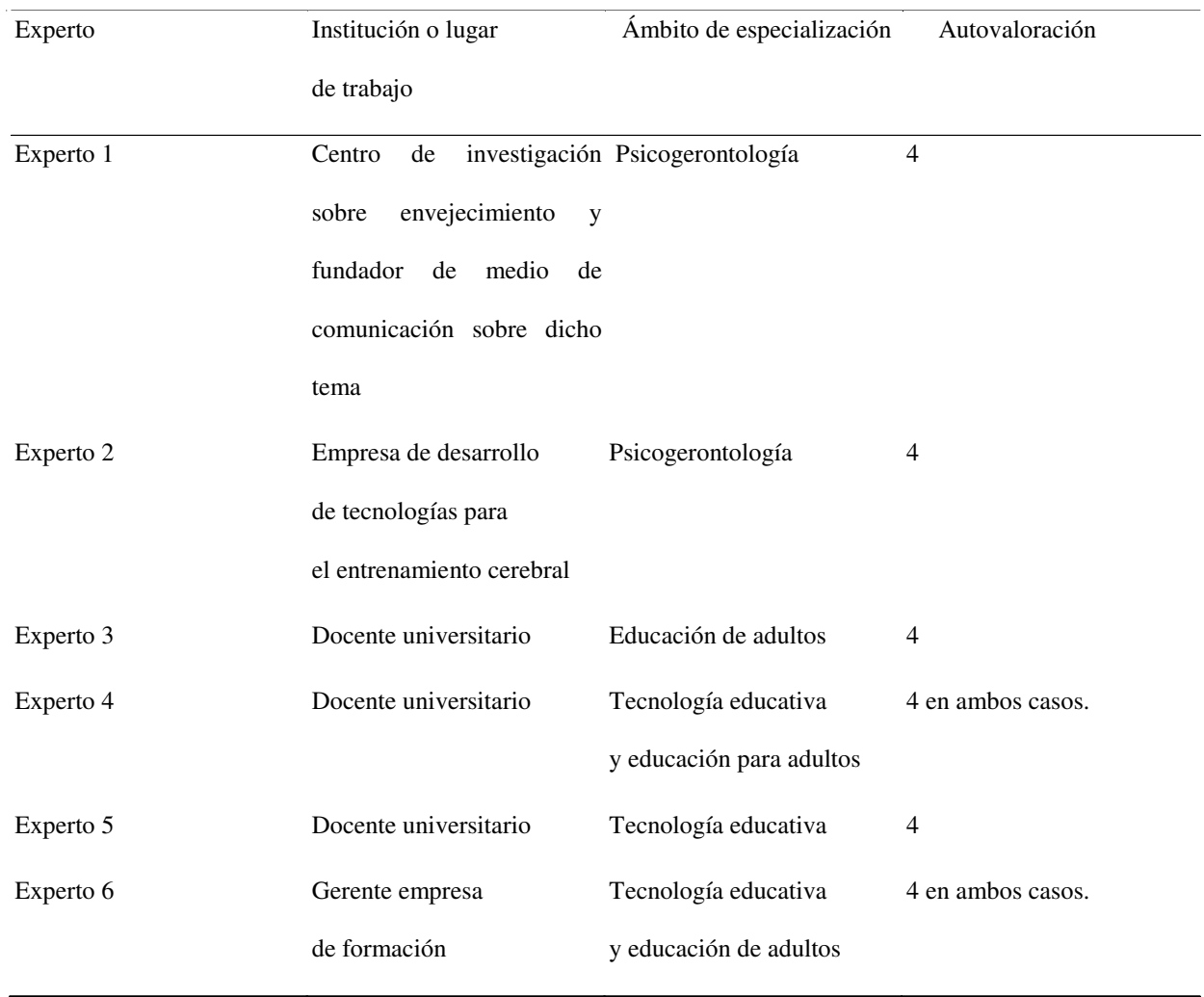

\section{Tabla 3. Características de los expertos consultados.}

sometidas a una o más re-evaluaciones por parte del mismo grupo de expertos. De esta forma, no se trata solamente de obtener opiniones, sino de desarrollar todo un proceso reflexivo sobre la posible secuencia de acontecimientos de la realidad estudiada.

En esta investigación se contó con la participación final de seis expertos, tres hombres y tres mujeres, vinculados al ámbito de las nuevas tecnologías en educación, la formación para adultos, y de la atención a personas mayores (Tabla 3). A cada uno de los expertos se le pidió que auto valorasen su nivel de pericia en el campo solicitado (tecnología educativa, educación para adultos o psicogerontología) en una escala de 1 a 5 , siendo 1 nada de pericia y 5 mucha pericia.

En un primer momento se envió una carta informativa del estudio, donde se solicitaba su colaboración a ocho expertos. Tras recibir sus respuestas, se envió a aquellos expertos que aceptaron la invitación, un resumen de la justificación del estudio así como del objetivo del mismo, junto con el cuestionario de opinión. Una vez recibidos sus cuestionarios, se hizo un análisis de contenido del mismo. A partir de dicho análisis se elaboró un primer borrador de resultados. Este borrador se utilizó como documento para preparar el segundo cuestionario donde los expertos 


\begin{tabular}{lllll}
\hline Año & Total & Hombres & Mujeres & Dif., hombres - mujeres \\
\hline 2006 & 32.6 & 39.6 & 25.7 & 13.9 \\
2007 & 37.9 & 42.7 & 33.2 & 9.5 \\
2008 & 42.5 & 49 & 35.9 & 13.1 \\
2009 & 47.5 & 53.3 & 41.8 & 11.5 \\
2010 & 53 & 57.5 & 48.5 & 9 \\
2011 & 57.3 & 60.9 & 53.6 & 7.3 \\
2012 & 60.8 & 65.6 & 56 & 9.6 \\
2013 & 63.4 & 67.3 & 59.4 & 7.9 \\
\hline
\end{tabular}

Tabla 4. Porcentaje de personas que han utilizado Internet al menos una vez por semana en los últimos 3 meses.

podían dar su opinión sobre los resultados preliminares, además de ofrecer nuevas aportaciones sobre el propio fenómeno en análisis. Una vez recibidos los segundos cuestionarios, se procedió de nuevo a un análisis de contenido. Esta segunda fase permitió la elaboración de un segundo borrador de resultados que también se envió al grupo de expertos. En esta segunda ocasión, los resultados fueron concordantes entre sí, de forma que el segundo borrador de resultados se redactó como informe final de dichos resultados. El cuestionario inicial incluía un total de siete siguientes preguntas abiertas.

\subsection{Análisis.}

Para satisfacer el objetivo de la investigación a partir de los datos procedentes del INE se realizó un análisis gráfico de la evolución a partir del porcentaje de cada año para cada ítem. Posteriormente se aplicaron distintos contrastes de hipótesis de proporciones entre 2006 y 2013, así como entre hombres y mujeres en 2006 y en 2013. El escaso número de datos de la secuencia histórica impide realizar un estudio de regresión. Los análisis se realizaron con el paquete Rstudio (2013).

Los informes aportados por el grupo de expertos se analizaron de forma cualitativa. Se realizó un análisis de contenido de las preguntas abiertas de los cuestionarios enviados a los expertos. El procedimiento se enriqueció con una triangulación entre los investigadores, que realizaron un análisis inicial de forma independiente. Posteriormente, los investigadores mantuvieron una reunión de puesta en 


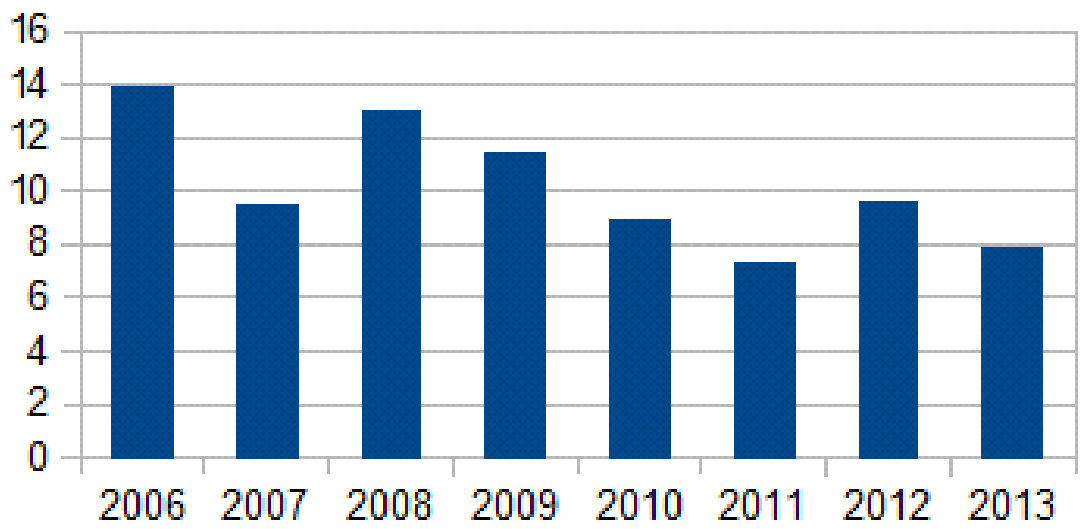

Gráfico 1. Diferencias de porcentajes de uso de internet entre hombres y mujeres.

\begin{tabular}{lllll}
\hline Año & Total & Hombres & Mujeres & Dif., hombres - mujeres \\
\hline 2006 & 7.7 & 9.8 & 5.6 & 4.2 \\
\hline 2007 & 11.6 & 12.8 & 10.4 & 2.4 \\
2008 & 11.4 & 13.1 & 9.7 & 3.4 \\
2009 & 13.3 & 16.8 & 9.7 & 7.1 \\
2010 & 14 & 17.3 & 10.7 & 6.6 \\
2011 & 16.2 & 18.6 & 13.8 & 4.8 \\
2012 & 19.6 & 22.2 & 16.9 & 5.3 \\
2013 & 20.5 & 23.2 & 17.9 & 5.3 \\
\hline
\end{tabular}

Tabla 5. Porcentaje de personas que han comprado a través de Internet en los últimos 3 meses. 


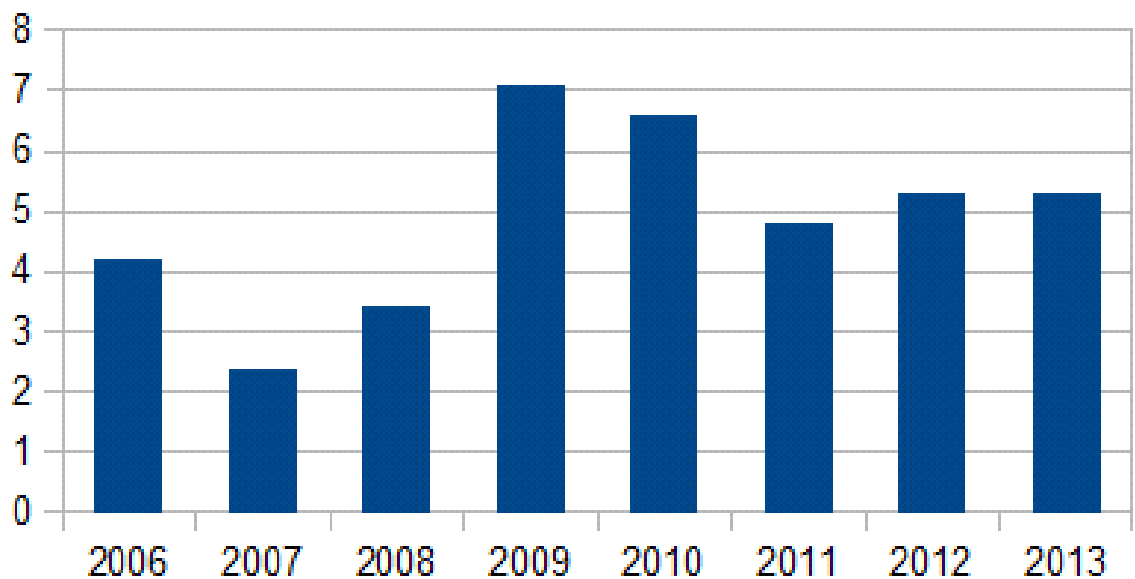

Gráfico 2. Diferencias de porcentajes de compras por internet entre hombres y mujeres.

común. Estos procesos se repitieron en las dos oleadas de informes. Finalmente, el grupo de investigadores fue el encargado de redactar el informe final de resultados con relación a las evaluaciones del panel de expertos.

\section{Resultados.}

\subsection{Evolución anual del uso de internet.}

Los datos de la encuesta muestran un aumento progresivo del uso habitual de internet (Tabla 4). Si en el año 2006 el porcentaje de uso se situaba en el $32.6 \%$ de los ciudadanos consultados, en el año 2013 el porcentaje aumentó hasta el $63.4 \%$. Este aumento fue significativo según el contraste de hipótesis (Chi-cuadrado $=1194663 ;$ g.l. $=1$; $\mathrm{p}<.0001)$.

Como se puede observar en el Gráfico 1, las diferencias entre hombre y mujeres presentan una forma de dientes de sierra. Es decir, un año con una elevada diferencia, da paso a una disminución para volver a aumentar posteriormente. A pesar de este proceso de altos y bajos, la tendencia general es a la reducción de las diferencias entre hombres y mujeres en el uso habitual de internet. Así, mientras que la diferencia en 2006 era de $13.9 \%$ a favor de los hombres, en 2013 dicho porcentaje se redujo al 7.9\% (Tabla 4). En cualquier caso, las diferencias entre hombres y mujeres siguen siendo significativas a un nivel de confianza superior al 99\% (Chi-cuadrado=46635.21; g.l.=1; $\mathrm{p}>0.001$ ).

Con relación al porcentaje de personas que compran por internet, destaca el relativo bajo porcentaje en comparación al porcentaje de personas que usan internet. No obstante, el porcentaje de compras también se ha visto incrementado en la secuencia histórica de forma progresiva (Tabla 5). La diferencia entre 2006 y 2013 fue significativa a un nivel de confianza del 99\% (Chi-cuadrado= 411471; g.l. $=1 ; \mathrm{p}<0.0001)$.

Con relación a la diferencia entre hombres y mujeres, de nuevo se registra un uso mayor 
por parte de los hombres en todos los años. Esta diferencia parece estabilizarse en los últimos 4 años, después de una disminución de los años 2007 y 2008, que fue abruptamente interrumpida en el año 2009 (Gráfico 2). Las diferencias entre hombres y mujeres sigue siendo significativa en 2013 (Chi-cuadrado= 29845.12; g.l.=1; p<0.0001) tal como lo era en 2006 (Chi-cuadrado= 35783.92; g.1.= 1; $\mathrm{p}<=0.0001)$.

\subsection{Procedimiento Delphi: Valoración de los expertos.}

Con relación a si la tecnología que usará en el futuro el segmento de población mayor está ya inventada, los expertos concluían que la tecnología actual estará presente aunque experimentará un desarrollo considerable (desarrollo de la interconectividad, aplicaciones específicas, usos nuevos a lo que ya existe, etc.). No obstante, los expertos también consideran que aparecerán nuevas tecnologías, algunas de las cuales son impensables hoy en día (Cuadro 1).

De forma unánime, los expertos consideran los recursos tecnológicos útiles para un envejecimiento activo (Cuadro 2). En su opinión, las tecnologías lo favorecerán jugando diversos roles:

Exigiendo a la persona una actualización continua para utilizar las nuevas tecnologías (que vayan surgiendo), especialmente si están adaptadas tanto en contenido como en funcionalidad y formas de uso.

Como medio para acceder a recursos y bienes tanto sociales como educativos como institucionales y económicos.

Como recurso para realizar un seguimiento de la salud de las personas.
En la tercera pregunta se les pidió a los expertos una previsión sobre el tipo de tecnología o qué características tendrá esa tecnología en el futuro. Aunque es simplemente una previsión, hubo unanimidad en afirmar que tendrá las siguientes características:

Se basará y diseñará pensando en la interconectividad entre equipos y la conexión a través de la red (internet).

Existirá una diversidad de equipos con funciones similares (tablets, ordenadores, móviles, y otros aún por inventar).

Se diseñará pensando en que pueda ser usada en cualquier lugar, primando la movilidad.

Igualmente, se destaca que serán recursos con un diseño amigable, fáciles de usar, con funciones adaptadas a las características de cada persona, cuyo fin será facilitar la independencia de las personas, su entrenamiento y su monitorización (Cuadro 3).

En opinión de los expertos la tecnología aportará diversas mejoras a la calidad de vida de las personas mayores: orientar, monitorizar, aprender, mantenimiento y promoción de las relaciones sociales, y facilitar inclusión social entre otras ventajas (Cuadro 4). Estas mejoras pueden agruparse en base a dos ejes:

\section{- Salud física y psicológica.}

Relaciones sociales.

En quinto lugar se preguntó a los expertos si consideraban la población española actual de 45 a 54 años tiene un nivel de formación «aceptable» en tecnología en función a los datos del INE. En general la opinión es que la formación es aceptable pero que no es suficiente para el futuro.

Se señala que la utilización de los teléfonos o internet (además de tecnologías más clásicas como televisión) está generalizada porque se 
Cat. A. Experto 1. "Año a año experimentamos novedades dentro de las herramientas que ya utilizamos".

Cat. A. Experto 2. "Por otro lado, y en relación a ese mismo desarrollo, creo que es muy posible que en 20 años dispongamos de nuevos dispositivos totalmente distintos a los que utilizamos en el momento presente".

Cuadro 1. Ejemplo de las opiniones de los expertos a la pregunta 1.

Cat. A. Experto 5. "Sí, mantienen la actividad y proporcionan destrezas y habilidades que permiten mantenerse activos".

Cat. A3. Experto 2. "Creo que la tecnología tiene distintas formas de favorecer el envejecimiento activo y la calidad de vida, por ejemplo: Promueve un aprendizaje continuo en sí mismo, aprender a manejar los dispositivos, moverse en intemet, manejar programas específicos, es ya una vía de aprendizaje".

Cuadro 2. Ejemplo de las opiniones de los expertos a la pregunta 2.

Cat. A3. Experto 4.

" Intuitivas (amigables)

- Interactivas

De bajo coste."

Cat. A1.1. Experto 2. "El uso de intemet en cualquier dispositivo también está claro, porque permite acceder a muchos recursos de aprendizaje y a nivel de relaciones sociales".

Cuadro 3. Ejemplo de las opiniones de los expertos a la pregunta 3.

Cat. A3.1. Experto 2. "Promoción del aprendizaje y con ello una mayor reserva cognitiva de cara a la salud cerebral. Acceso a recursos sociales y establecimiento de redes sociales más amplias, reduciendo así el aislamiento social. Acceso a más recursos sanitarios, favoreciendo una mejor monitorización de personas en domicilio y en zonas rurales. Acceso a recursos intelectuales y de ocio."

Cuadro 4. Ejemplo de las opiniones de los expertos a la pregunta 4. 
Cat. B. Experto 3. "En principio puede considerarse aceptable aunque no es suficiente y habrá que potenciar el uso eficiente de esta tecnología. A pesar del boom de los primero años en su implantación quizá convenga considerar que hay que seguir "alfabetizando" a la población adulta." Cat. A3.1. Experto 4. " ... creo también que, a partir de los datos ofrecidos, es difícil conocer el nivel de la formación de este grupo (el nivel de acceso no es sinónimo de calidad de uso). Los marcos temporales de utilización de la tecnología (3 meses, una vez a la semana) - tampoco permiten aclarar si el nivel de formación es 'aceptable'."

Cat. B. Experto 5. "Considero que en ese rango de edad la formación es aceptable."

Cuadro 5. Ejemplo de las opiniones de los expertos a la pregunta 5.

Cat. B. Experto 4. "Hacen falta distintos tipos de intervención y de formación:

1. En primer lugar, hay que plantear la resolución de los problemas de la desigualdad de acceso.

2. Una vez garantizado el acceso, es fundamental pensar en los usos y en distintos grados de alfabetización digital - desde las competencias instrumentales a las competencias de ciudadanía y de participación digital"

Cuadro 6. Ejemplo de las opiniones de los expertos a la pregunta 6.

Cat. D. Experto 4. "Veo complicado que las administraciones se impliquen en la formación de personas con este rango de edad, aunque debería ser tenerse en cuenta."

Cuadro 7. Matización sobre la pregunta 6 en la segunda oleada.

ha producido una implementación progresiva de los mismos entre la sociedad, dando tiempo a su aprendizaje. Igualmente, se subraya también que las estadísticas del INE se centran en un período de tiempo (3 meses) que puede no recoger bien la realidad (Cuadro 5).

Con relación a la sexta pregunta del cuestionario (¿Piensa usted que la población actual de 45 a 54 años necesitará de una formación específica en tecnología para el uso eficiente de la tecnología futura?) los expertos concluyen que la formación actual puede ser suficiente para la tecnología de ahora, y que la formación ha sido fundamentalmente autodidacta (Cuadro 6). Probablemente, según los expertos consultados, en el futuro la formación autodidacta no será suficiente, lo que obligará a establecer sistemas de formación específica y con un carácter híbrido, esto es, empleando todo tipo de medios y recursos didácticos virtuales y presenciales, individuales y en red, formales y no formales. El medio de llevar a cabo esta formación puede ser a través de cursos, folletos, campañas en los medios de 
comunicación, redes sociales, formación comunitaria, etc.

Los expertos sugieren que estas actuaciones deberían estar gestionadas o controladas por las instituciones, principalmente los servicios públicos. En la segunda oleada de consulta, algunos de los expertos manifestaban su escepticismo sobre la implicación efectiva de la administración pública para ofrecer planes de formación eficientes (Cuadro 7).

A colación de esta pregunta, el grupo de expertos apuntó algunas cuestiones a tener en cuenta:

Antes de establecer planes formativos es necesario superar la actual brecha entre los que tienen acceso y los que no tienen acceso a la tecnología.

Es necesario comprobar la confianza en los recursos tecnológicos actuales, más allá del simple uso de los mismos.

\section{Discusión y conclusiones.}

Tanto los datos del INE, como la opinión del panel de expertos coinciden en mostrar un aumento progresivo en el uso de internet en la vida cotidiana. Estos resultados son coincidentes con otros estudios, como el World Internet Project Spain 2013 (Aramda, Sánchez \& Tubella, 2014) donde se estimó un aumento del uso de internet de 15 puntos entre 2011 y 2013.

Los datos del INE también ponen de manifiesto que si bien hombres y mujeres tienden a unificar sus hábitos de uso de internet, se mantienen las diferencias en el momento de comprar por internet. Este resultado coincide con estudios previos, donde se ha puesto de manifiesto la brecha entre hombres y mujeres (Observatorio eigualdad, 2011; Ministerio de Sanidad,
Servicios Sociales e Igualdad, 2012). En este sentido, se podría hipotetizar que las mujeres tendrían una menor confianza en la seguridad de internet para hacer gestiones complejas, o bien que prefieren la interacción directa para realizar las compras que tienen un mayor componente relacional.

Será necesario profundizar en las razones del distanciamiento entre hombres y mujeres, puesto que aunque es razonable un uso diferencial, no se entiende un distanciamiento cuantitativo, y menos aún que este se mantenga año tras año.

Los expertos coinciden que la tecnología futura está aún por inventar, si bien ésta será resultado de una evolución natural a partir de la existente. Esta tendencia coincide con las estimaciones realizadas por otros autores, quienes además sitúan a la innovación como un elemento clave de la evolución de las TIC (Martínez Ruiz, Martínez Salgado, Allberich \& Poyatos, 2013). Dentro de esta evolución, el seguimiento y monitorización médica es uno de los campos que se verá potenciado por la tecnología. Aunque este campo de aplicación es una realidad (Ramos et al., 2013) el panel de expertos sugiere que en el futuro inmediato experimentará un incremento destacado, lo que coincide con las conclusiones de otros estudios (García Lizana, 2013).

Con relación a la formación, el panel de expertos sugiere que en el futuro será necesario que la persona asuma un papel activo en aprender a utilizar la nueva tecnología para favorecer la calidad de vida durante su envejecimiento. Estas conclusiones han sido puestas de manifiesto por otros estudios (Ministerio de Sanidad, Servicios Sociales e Igualdad, opus cit.). Además, en estos estudios, se hace hincapié en la necesidad de actuar específicamente 
sobre la brecha entre géneros. El panel de expertos ha puesto de manifiesto la necesidad de actuar también sobre la brecha social y económica para posibilitar el acceso a las TIC a toda la ciudadanía. Actuaciones que deben ser emprendidas por las administraciones públicas como una forma de servicio a sus ciudadanos. Así, las acciones formativas para toda la ciudadanía en materia de alfabetización digital, se convierten en elementos básicos e ineludibles para promover la equidad y la cohesión social en una sociedad como la actual. Si hoy se puede hablar con certidumbre de nuevos marcos relacionales digitales, así como de emergentes formas de socialización influenciadas y netamente impregnadas del mundo digital, no resulta coherente desasistir, desde un punto de vista formativo comunitario, a una parte de la población, que año a año va aumentando en número y potencial sociocomunitario.

Con relación al primer objetivo parcial de este estudio, los resultados muestran un grado de penetración de las tecnologías que puede calificarse de medio en la población de 45 a 54 años. Aunque parece aumentar su utilización cotidiana, su utilización en operaciones comerciales es bastante bajo. Esto apunta a que se mantiene la tendencia al comercio tradicional, pero también a una posible desconfianza en las operaciones monetarias por internet.

Por su parte, el panel de expertos apunta que las TIC en sí mismas beneficiarán a la población mayor en tres aspectos básicos:

Motor de aprendizaje continúa. La renovación continúa de los artilugios tecnológicos, junto con la implicación de los ciudadanos en su utilización, motivarán a los propios ciudadanos para continuar aprendiendo el uso de dichos artilugios. En este sentido se comprende que las TIC sean un factor para el aprendizaje a lo largo de la vida.

Mantenimiento de las relaciones sociales. La conectividad, basada en la red Internet, será posible desde distintos artilugios, algunos ya disponibles, y otros por venir. A esto habrá que añadir la personalización de los dispositivos para una utilización mucho más efectiva. Todo ello afectará positivamente al mantenimiento de las redes sociales aunque sea a costa de cambiar el soporte básico de las mismas.

Monitorización médica y psicológica. Actualmente el desarrollo de sistemas de seguimiento está experimentando un destacado desarrollo tal como se expuso anteriormente. En el futuro se espera que este campo evolucione rápidamente.

Respecto al segundo objetivo del estudio, el grupo de expertos apunta la necesidad de que las personas de 45 a 54 años establezcan plantes de formación, asumiendo su propia responsabilidad en ello, para poder integrar la tecnología como un recurso más para poder tener una mejor calidad de vida en el futuro. Estos planes, que requieren de un alto grado de autonomía en su definición y ejecución, no pueden estar exentos de la necesaria supervisión y orientación por parte de instituciones, instancias o plataformas virtuales que puedan ofrecer una cobertura formativa de calidad para adultos que quieren aprovechar al máximo las oportunidades que les ofrece el mundo tecnológico y en red para su crecimiento y bienestar personal, social.

En definitiva, aunque la formación en el uso de las TIC en este segmento de la población parece adecuada en la actualidad, en el futuro deberá aprender a utilizar unas nuevas tecnologías que será difícil de forma autodidacta, por su complejidad y por su velocidad de desarrollo. 


\section{Referencias bibliográficas.}

Aldana González, G., García Gómez, L, \& Jacobo Mata, A. (2012). Las TIC como alternativa par la estimulación de los procesos cognitivos en la vejez. CPU-e, Revista de investigación educativa, 14.

Aranda, D., Sánchez, J., \& Tubella, I. (2014). World internet project Spain 2013. Informe de resultados. Barcelona: UOC. Recuperdo de http://hdl.handle.net/10609/31701

Recuperado de http://www.uv.mx/cpue/

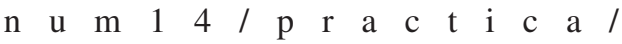
aldana_garcia_mata_tic_vejez.html

Berner, J., Rennemark, M., Jogréus, C. \& Berglund, J. (2012). Distribution of personality, individual characteristics and internet usage in Swedish older adults. Aging \& mental health, 16 (1), 119-126. doi: 10.1080/ 13607863.2011.602958

Berner, J.S., Rennemark, M., Jogréus, C. \& Berglund, J. (2013). Factors associated with change in Internet usage of Swedish older adults (2004-2010). Health informatics journal, 19 (2), 152-162. doi: 10.1177/ 1460458212462151

Burmeister, O.K. (2010). Websites for seniors: cognitive accessibility. International Journal of Emerging Technologies and Society, 8 (2), 99-113.

Castaño, J., Redecker, C., Vuorikari, R. \& Punie, Y. (2013). Open Education 2030: planning the future of adult learning in Europe. Open Learning: The Journal of Open, Distance and e-Learning, 28 (3), 171186. doi:10.1080/02680513.2013.871199

García Aretio, N. (2007) (Coord.). De la educación a distancia a la educación virtual. Barcelona: Ariel.

García Lizana, F. (2013). Cooperación para la innovación europea en el envejecimiento activo y saludable: de la política a la acción. Gaceta Sanitaria, 27 (5), 459-462.

González, M.G.A., Gómez, M.L.G. \& Mata, A.J. (2012). Las Tecnologías de la Información y Comunicación (TIC) como alternativa para la estimulación de los procesos cognitivos en la vejez. CPU-e, Revista de Investigación Educativa, 14, 153-166.

Grzesiak, M. \& Richert-Ka Ÿmierska, A. (2013). Educational engagement of the elderly-the experiences of selected Baltic Sea Region countries. Prace Naukowe Uniwersytetu Ekonomicznego we Wroc $^{3}$ awiu, 286, 133-143.

IMSERSO (2012). Informe 2010. Las personas mayores en España. Datos estadísticos estatales y por Comunidades Autónomas, I. Madrid: IMSERSO http:// www.imserso.es/InterPresent1/groups/ i m s e r s o / d o c u m en t s/bin a rio/ 22023_inf2010pm_v1.pdf

Luque, L.E. (2007). Estimulación cognitiva mediante recursos informático. Revista de la Asociación colombiana de Gerontología y Geriatría, 21 (4), 1093-1098.

Martínez Ruiz, I., Martínez Salgado, M., Allberich, A.\& Poyatos, J.M. (2013). Tendencias a futuro. BIT, 195, 64-67. Recuperado de http://www.coit.es/ p u b l i c a c i ones/bit/bit $195 /$ monografico6_195.pdf

Observatorio e-Igualdad UCM (2011). La brecha digital de género en España: análisis multinivel (España, Europa, CCAA). Madrid: Ministerio de Sanidad, Servicios Sociales e Igualdad.

Ojeda, G. (2008). Brecha y alfabetización digital en la formación ciudadana. En J.M. García de Madariaga, J.C. Calvi, F. Trucho y M. Meda (Eds.), Políticas de comunicación en España y Latinoamérica. Medios 
convencionales, Tercer Sector Audiovisual y Alfabetización digital. Madrid: Dykinson.

Ontsi (2013). Informe Recopilatorio 20042012, las Tic en los Hogares Españoles. Recuperado de http://www.ontsi.red.es/ontsi/ es/estudios-informes/informe-recopilatorio2004-2012-las-tic-en-los-hogaresespa\%C3\%B1oles

Ortoll, E. (Coord.) (2007). La alfabetización digital en los procesos de inclusión social. Barcelona: UOC.

Punie, Y. \& Ala-Mutka, K. (2007). Future Learning Spaces: new ways of learning and new digital skills to learn. Nordic Journal of Digital Literacy, 2 (4), 210-225.

Ramos, V., Arce, J.L., Fanjut, F., Arambarri, J., Rodal, J, Jiménez, F. \& Guerra, P. (2013). Ventajas de la aplicación de las TIC a la salud. BIT, 195, 46-49. Recuperado de http:// www.coit.es/publicaciones/bit/bit195/ monografico2_195.pdf

Regan, E.A. (1998). Lifelong learning and performance: Linking academia and Business. Office Systems Research Journal, 16(2), 4353.

Rosenberg, D., Depp, C.A., Vahia, I.V., Reichstadt, J., Palmer, B. W., Kerr, J. \& Norman, G. (2010). Exergames for subsyndromal depression in older adults: a pilot study of a novel interaction. American Journal of geriatric psychiatry, 18 (3). Recuperado de http://www.helmholtzmuenchen.de/fileadmin/JOIN/PDF/ PID1790829-Exergames.pdf doi: 10.1097/ JGP.0b013e3181c534b5

Rstudio (2013). Rstudio Version 0.98.987. RStudio, Inc.

Salanova, M. \& Peiró, J.M. (Eds.)(2001). Nuevas tecnologías y formación continua en la empresa: un estudio psicosocial. Castelló: Universidad Jaume I.
Sebastián Morillas, S. \& Martínez Navarro G (2013). La influencia de las nuevas tecnologías: videojuegos, redes sociales e internet, en los consumidores seniors en España. Actas del I Congreso Internacional Comunicación y Sociedad. Logroño: Universidad Internacional de la Rioja

Shubert, T.E. (2010). The use of commercial health video games to promote physical activity in older adults. Annals of long-term care, 18(5), 27-32.

Tam, M. (2013). Perceptions of successful ageing and implications for late-life learning. Studies in continuing education, 35 (3), 281298. doi: http://dx.doi.org/10.1080/ 0158037X.2012.746228

Tam, M. (2014a). Understanding and Theorizing the Role of Culture in the Conceptualizations of Successful Aging and Lifelong Learning. Educational Gerontology, 40 (12), 881-893. doi:10.1080/ 03601277.2014.907072

Tam, M. (2014b). Intergenerational Service Learning Between the Old and Young: What, Why and How. Educational Gerontology, 40 (6), 401-413.

Tójar, J.C. (2006). Investigación cualitativa. Comprender y actuar. Madrid: La Muralla.

Fecha de recepción: 18-02-2015

Fecha de evaluación: $05-05-2015$

Fecha de aceptación: 22-05-2015 